\title{
Fangchinoline attenuates cardiac dysfunction in rats with endotoxemia via the inhibition of ERK1/2 and NF-KB p65 phosphorylation
}

\author{
Hongwei Chen ${ }^{1}$, Zonghua Shi ${ }^{2}$, Yongsheng Xing ${ }^{1}$, Xinwei $\mathrm{Li}^{3}$, Fengzhou Fu ${ }^{4}$ \\ ${ }^{1} \mathrm{CCU}$, Xinxiang Central Hospital, Xinxiang, China; ${ }^{2} \mathrm{CCU}$, The Fifth Affiliated Hospital of Zhengzhou University, Zhengzhou, China; ${ }^{3}$ Department \\ of Basic Medicine, Louhe Medical College, Louhe, China; ${ }^{4}$ Department of Cardiology, Dongguan Songshan Lake Central Hospital, Dongguan, \\ China \\ Contributions: (I) Conception and design: H Chen, Y Xing; (II) Administrative support: H Chen, Z Shi; (III) Provision of study materials or patients: \\ H Chen, Z Shi, X Li, F Fu; (IV) Collection and assembly of data: All authors; (V) Data analysis and interpretation: H Chen, Y Xing, X Li, F Fu; (VI) \\ Manuscript writing: All authors; (VII) Final approval of manuscript: All authors. \\ Correspondence to: Yongsheng Xing. No. 56 Jinsui Avenue, Xinxiang, Henan, China. Email: xinxiangchw@163.com.
}

\begin{abstract}
Background: Cardiac dysfunction is a complication commonly encountered by patients with endotoxemia. Fangchinoline (Fan) is a natural bisbenzylisoquinoline alkaloid. This study aimed to investigate the cardioprotective effect of Fan against lipopolysaccharide (LPS)-induced acute cardiac dysfunction.

Methods: Rats were administered with Baicalin $(100 \mathrm{mg} / \mathrm{kg})$ and Fan $(30$ or $60 \mathrm{mg} / \mathrm{kg}$ ) via intraperitoneal injection (i.p.) for 3 days, followed by LPS treatment $(10 \mathrm{mg} / \mathrm{kg}$, i.p.). The rats were randomly grouped $(\mathrm{n}=10)$ : the control group, the LPS group, the LPS + Baicalin group, the LPS + Fan groups. Echocardiography and hematoxylin and eosin (HE) staining were performed to detect cardiac dysfunction. Cardiac function were also determined by quantitative reverse transcription-polymerase chain reaction (qRT-PCR), ELISA, and western blot, respectively. The protective mechanisms of Fan were analyzed by western blot and qRT-PCR.

Results: LPS induced the depression of cardiac function, myocardial inflammation, and apoptosis. These changes were associated with decreased GRP78 and GADD34, increased C/EBP-homologous protein (CHOP) and cleaved caspase-12. Fan significantly reduced the release of inflammatory cytokines such as monocyte chemotactic protein-1 (MCP-1), tumor necrosis factor- $\alpha$ (TNF- $\alpha$ ), interleukin (IL)-1 $\beta$, IL-18, and IL-6. Furthermore, Fan treatment increased superoxide dismutase (SOD) and decreased malondialdehyde (MDA. Notably, Fan inhibited myocardial apoptosis following ER stress in the LPS-induced rat model and stimulated phosphorylation activation of ERK1/2 and NF- $\mathrm{B}$ p 65 proteins.
\end{abstract}

Conclusions: Fan deficiency alleviated LPS-induced endotoxemia in rats. Therefore, Fan may be a new therapeutic approach for the treatment of cardiac dysfunction.

Keywords: Fangchinoline (Fan); endotoxin; myocardial inflammation; endoplasmic reticulum stress (ER stress)

Submitted Jul 15, 2020. Accepted for publication Sep 02, 2020.

doi: $10.21037 /$ atm-20-5669

View this article at: http://dx.doi.org/10.21037/atm-20-5669

\section{Introduction}

Severe sepsis are the main causes of morbidity and mortality in patients with cardiac dysfunction $(1,2)$. Patients with sepsis can experience multiple organ failure and early myocardial dysfunction caused by dysregulated immunologic and inflammatory response (3). Approximately
$45 \%$ of patients with sepsis develop myocardial dysfunction, which could lead to the deterioration of heart function and, consequently, poor prognosis (4). The myocardial system plays a pivotal role in the pathophysiology of septic shock, organ failure, and death (5). Myocardial dysfunction in sepsis has multiple complex manifestations, including 
decreased ejection fraction and myocardial contractility. Additionally, previous studies have shown that the presence of acute cardiac dysfunction in sepsis is an indicator of poor prognosis $(6,7)$.

Cardiac dysfunction is a complication frequently encountered by patients with endotoxemia, and it has also been observed in murine models of lipopolysaccharide (LPS)-induced sepsis $(8,9)$. LPS is located in the outermost layer of the cell wall of gramnegative bacteria and has a considerable influence on cardiac function (10). Other studies have shown that LPS-induced cardiac complications are associated with cardiac apoptosis (11), as well as over-expression of proinflammatory cytokines (12). Furthermore, Tocmo and Parkin (13) also reported that LPSinduced activation of proinflammatory cytokines was triggered by nuclear factor- $\mathrm{\kappa B}(\mathrm{NF}-\kappa \mathrm{B})$, which could stimulate the overexpression of multiple proinflammatory cytokines, thereby affecting myocardial function. In contrast, NF- $\mathrm{BB}$ inactivation has been shown to attenuate LPS-induced cardiac dysfunction $(14,15)$. Nevertheless, the underlying mechanism of sepsis with endotoxemic cardiac dysfunction has yet to be fully illuminated.

Traditional Chinese medicinal herbs have been widely used in the treatment of various diseases. Fangchinoline (Fan) is a natural bisbenzylisoquinoline alkaloid isolated from Stephaniae tetrandra S. Moore. Previous studies have demonstrated that Fan could inhibit cell proliferation and/ or apoptosis to modulate the development of multiple cancers, including bladder cancer (16), lung cancer (17), and hepatocellular carcinoma (18). Moreover, Fan is also involved in strong free radical scavenging, antihyperglycemic, anti-oxidant, and anti-thrombotic activity $(19,20)$. Thus, Fan shows great medicinal potential for the treatment of several disorders. Nevertheless, the pathological mechanism underlying the protective effect of Fan against septic cardiac dysfunction remains poorly understood, which requires further studies.

In this study, we evaluated the anti-apoptosis and antiinflammatory effects of Fan on acute cardiac dysfunction in an LPS-induced rat model, and explored the potential protective mechanism.

We present the following article in accordance with the ARRIVE reporting checklist (available at http://dx.doi. org/10.21037/atm-20-5669).

\section{Methods}

\section{Main materials}

Fan $\left(\mathrm{C}_{37} \mathrm{H}_{40} \mathrm{~N}_{2} \mathrm{O}_{6}\right.$, molecular weight: 608.72 , purity
$>98 \%)$ was purchased from Shanghai Guandao Biological Engineering Co. Ltd. (Shanghai, China). LPS lyophilized powder was purchased from Sigma-Aldrich (St. Louis, Missouri, USA).

\section{Animal model and treatments}

The protocols of the animal experiments in this study were approved by the ethical committee of Xinxiang Central Hospital (ZXYY-KY-0058), and were conducted according to the published Guide for the Care and Use of Laboratory Animals (National Academies Press, 2011). Six-week-old male Wistar rats (weight: $120 \pm 2 \mathrm{~g}$ ) were obtained from the Institute of Zoology, Chinese Academy of Sciences (Beijing, China) and kept in a suitable environment $\left(22 \pm 3{ }^{\circ} \mathrm{C}\right.$, humidity $\left.55 \% \pm 5 \%\right)$ under a 12-hour light/dark cycle. The rats were given free access to food and water. Subsequently, the rats were randomly divided into 5 groups (with 10 rats in each group), including the control group: equal volumes of sterile saline, the LPS group: $10 \mathrm{mg} / \mathrm{kg}$ LPS, Baicalin group (21): LPS + Baicalin $(100 \mathrm{mg} / \mathrm{kg})$ group, and the Fan groups $(20,22)$ : LPS + Fan (30 or $60 \mathrm{mg} / \mathrm{kg}$ ). Each group received daily intraperitoneal injections with the same volume of sterile saline for 3 consecutive days. Approximately 1 hour after the final administration of sterile saline injection, the LPS groups were intraperitoneally injected with LPS $(10 \mathrm{mg} / \mathrm{kg})$, as previously described (12). At 3 hours after LPS injection, the Fan groups were performed with different doses of Fan. The control mice were administered sterile saline only. All experiments were carried out 6 hours after LPS injection. At the designated time points $(6 \mathrm{~h})$ after LPS/sterile saline treatment, the rats were sacrificed with sodium pentobarbital $(150 \mathrm{mg} / \mathrm{kg})$, and samples of their heart tissue were stored at $-80^{\circ} \mathrm{C}$ for later experiments. All experiments were performed in triplicate.

\section{Echocardiography}

At 12 hours after LPS treatment, the rats were anesthetized by intraperitoneal injection of $1.5 \%$ isoflurane. The left ventricular (LV) function parameters were determined by transthoracic echocardiography using Vevo2 100 (VisualSonics, Ontario, Canada) and a $12 \mathrm{MHz}$ transducer, including LV ejection fraction (LVEF), LV fractional shortening (LVFS), LV wall thickness (LVWT), LV endsystolic volume (LVESV), LV end-diastolic volume (LVEDV), and LV systolic pressure (LVSP). The 
Table 1 The primer sequence of genes used in this study

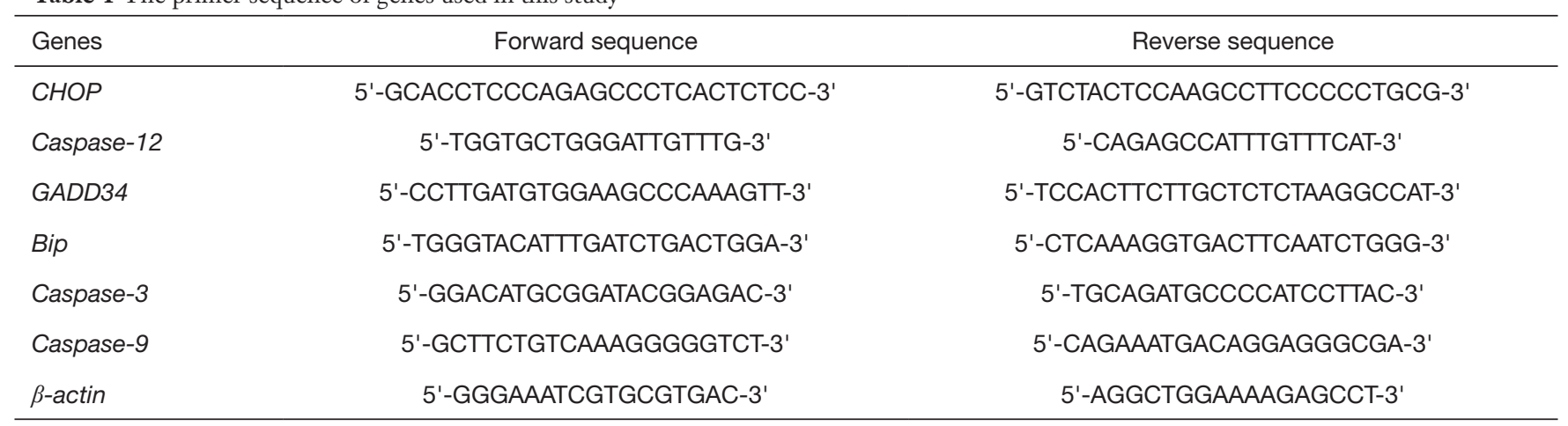

echocardiographic data were processed and the average from five consecutive cardiac cycles was calculated.

\section{Quantitative reverse transcription-polymerase chain reaction ( $q R T-P C R)$ analysis}

Briefly, total RNA was isolated from heart tissues with TRIzol reagent (Invitrogen, CA, USA). Then, iScript cDNA Synthesis Kit (Bio-Rad, CA, USA) was employed to reverse transcribe total RNA $(1 \mu \mathrm{g})$ to cDNA, according to the manufacturer's instructions. The subsequent amplification reaction was consistent with international standards. Table 1 lists the primer sequences used. All experiments were performed at least three times.

\section{Enzyme-linked immunosorbent assay (ELISA)}

The serum levels of MCP-1, TNF- $\alpha$, and IL-1 $\beta, 18$, 6, SOD, and MDA were determined using ELISA kits (Thermo Fisher, MA, USA), in line with the manufacturer's instructions.

\section{Immunobistochemistry (IHC) assay}

The heart tissues of the rats were fixed in $10 \%$ formalin, soaked in paraffin, and sliced into $4-\mu m$-thick sections. Next, the paraffinized sections were dewaxed and hydrated, and endogenous peroxidase activity was blocked with $3 \%$ $\mathrm{H}_{2} \mathrm{O}_{2}$ for 10 minutes. The sections were washed twice in phosphate-buffered saline (PBS) and then placed into $10 \mathrm{mM}$ hot citrate buffer for 10 minutes, before incubation with p-P65 antibody (ab86299, Abcam, Cambridge, UK) as a primary antibody overnight at $4{ }^{\circ} \mathrm{C}$. On the following day, the corresponding secondary antibodies were incubated at $37^{\circ} \mathrm{C}$ for 30 minutes. Finally, the sections were stained with a DAB kit (Beyotime, Haimen, China). A confocal microscope was used to capture images from a random field of vision (Leica, Wetzlar, Germany). All experiments were performed at least three times.

\section{Hematoxylin and eosin (HE) staining}

The steps are the same as previously described (12). The 4- $\mu \mathrm{m}$ sections of rat heart tissue were stained with HE. The counterstained tissue structures were examined using a confocal microscope (Leica Microsystems CMS GmbH, Wetzlar, Germany). All experiments were performed at least three times.

\section{TdT-mediated dUTP nick end labeling (TUNEL) staining}

The steps before dewaxing and hydration of paraffin sections were the same as that of IHC. After that, the sections were treated with DNase protease K (Thermo Fisher, MA, USA) for $30 \mathrm{~min}$. The TUNEL staining of these sections was performed using FragEL ${ }^{\mathrm{TM}}$ DNA Fragmentation Detection Kit (Merck, Billerica, USA). Five visible regions were randomly selected the under a microscope (Leica, Wetzlar, Germany) and the number of positive cells was counted.

\section{Western blot}

Proteins were extracted from the rat heart tissues using RIPA lysis buffer (Beyotime, Haimen, China). Protein concentrations were measured using a BCA protein assay kit (23227, Abcam). Post protein separation on $12 \%$ sodium dodecyl sulfate- polyacrylamide gel electrophoresis (SDS-PAGE), the separated protein was transferred to polyvinylidene fluoride (PVDF) membranes. The 
membranes were then blocked with $5 \%$ non-fat milk at $4{ }^{\circ} \mathrm{C}$ for 1 hour, and incubated with the following primary antibodies: DDIT3 antibody (ab10444, Abcam), cleaved caspase-12 antibody (\#2202, CST, MA, USA), GADD34 antibody (ab131402, Abcam), BiP antibody (ab21685, Abcam), cleaved caspase-3 antibody (ab184784, Abcam), cleaved caspase-9 antibody (\#9507, CST), IL-1 $\beta$ (205924, Abcam) p-ERK1/2 antibody (ab214362, Abcam), ERK1/2 antibody (ab17942, Abcam), P65 antibody (ab16502, Abcam), and p-P65 (phospho S536) antibody (ab86299, Abcam). After incubation overnight, the membranes were supplemented with the corresponding secondary antibodies. The bands were visualized with ECL reagent (Bio-Rad, CA, USA). All experiments were performed at least three times.

\section{Statistical analysis}

Data were analyzed and processed using SPSS 21.0 software (IBM, Armonk, USA). The data from each group were presented as mean \pm standard deviation (SD). Student's $t$-test was employed to evaluate significant differences between two groups, Significant differences between three or more groups were analyzed by one-way analysis of variance. A $\mathrm{P}$ value of $<0.05$ was considered to be statistically significant.

\section{Results}

\section{Fan prevented LPS- induced cardiac dysfunction in rats}

To assess whether Fan prevents LPS-induced cardiac dysfunction, the myocardial sections were stained with HE. As shown in Figure 1A, the control group showed no clear difference in cardiac morphology; however, in the LPS group, myocardial fiber fracture, cellular edema, and inflammatory cell infiltration were observed. Interestingly, 30 or $60 \mathrm{mg} / \mathrm{kg}$ Fan and $100 \mathrm{mg} / \mathrm{kg}$ Baicalin obviously attenuated myocardial tissue injury compared with LPS. Furthermore, we measured cardiac function in rats by echocardiography, 12 hours after the administration of LPS via intraperitoneal injection. As shown in Figure $1 B, C, D, E, F, G$ compared with the sterile salinetreated control group, the LPS-treated mice had increased LVESV but significantly decreased LVEF, LVFS, LVWT, LVEDV, and LVSP. However, the Fan and Baicalin groups showed remarkably improved cardiac function compared with the LPS-induced group.

\section{Fan effected LPS-mediated cardiomyocyte apoptosis following the ERS}

As shown in Figure 2A, LPS greatly up-regulated CHOP and cleaved caspase-12 at the mRNA level $(\mathrm{P}<0.05)$, compared with control; however, in the Fan (30 or $60 \mathrm{mg} / \mathrm{kg})$ and Baicalin $(100 \mathrm{mg} / \mathrm{kg})$ groups, the levels of CHOP and cleaved caspase-12 were significantly decreased $(\mathrm{P}<0.05)$, compared with LPS. Next, we further examined whether the protein levels of these markers also followed the trend. As shown in Figure 2B, LPS greatly up-regulated the protein levels of CHOP and cleaved caspase-12 $(\mathrm{P}<0.05)$, while Fan or Baicalin significantly decreased CHOP and cleaved caspase-12 expression $(\mathrm{P}<0.05)$. Also, we examined the expression of apoptosis-related genes (GADD34 and Bip). As shown in Figure 2C,D, LPS greatly down-regulated GADD34 and BiP expression $(\mathrm{P}<0.05)$. In contrast, Fan and Baicalin significantly increased the expression of GADD34 and BiP compared with LPS group $(\mathrm{P}<0.05)$.

\section{Fan blocked LPS-induced apoptosis and oxidative stress.}

As shown in Figure $3 A, B$, the apoptosis rate of myocardial tissues was examined by TUNEL assay. We founf that the apoptosis rate was significantly increased after LPS induction. Counterly, Fan (30 or $60 \mathrm{mg} / \mathrm{kg}$ ) or Baicalin $(100 \mathrm{mg} / \mathrm{kg})$ treatment reduced the apoptosis rate of myocardial tissues. Additionally, the protein levels of the markers of apoptosis (Caspase-3 and Caspase-9) were also detected. As shown in Figure 3C, compared with the control group or the LPS group, the protein levels of Caspase-3 and Caspase-9 were significantly decreased in the LPS + Fan or LPS + Baicalin group $(\mathrm{P}<0.05)$. Furthermore, the serum levels of SOD and MDA were also examined. The data showed that SOD decreased and MDA increased after LPS treatment when compared with control, but SOD was elevated and MDA was mitigated with Fan or Baicalin treatment compared with the LPS group (Figure 3D,E).

\section{Fan inbibited LPS-induced inflammatory response}

To evaluate the role of Fan in inflammation, the expressions of the above inflammatory cytokines were analyzed. As shown in Figure 4, LPS remarkably up-regulated the protein levels of MCP-1, TNF- $\alpha$, IL-1 $\beta$, IL-18, and IL-6, compared with control group $(\mathrm{P}<0.05)$. In contrast, in the Fan and Baicalin groups, the protein levels of these 
A
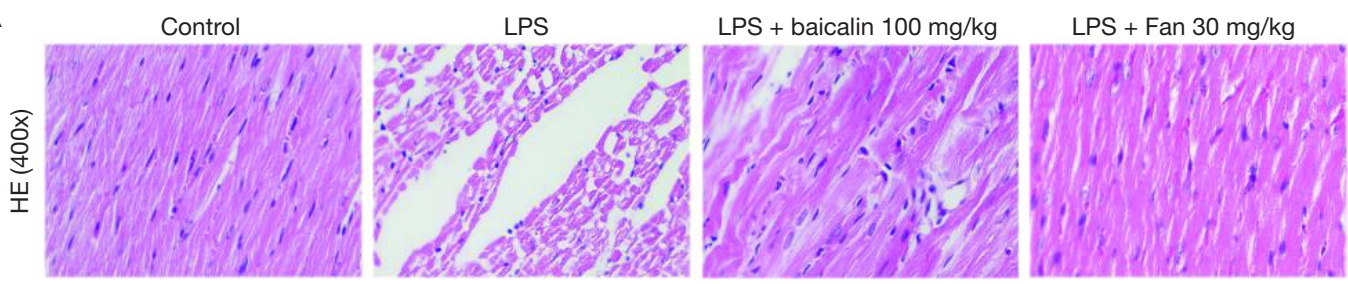

LPS + Fan $60 \mathrm{mg} / \mathrm{kg}$

B

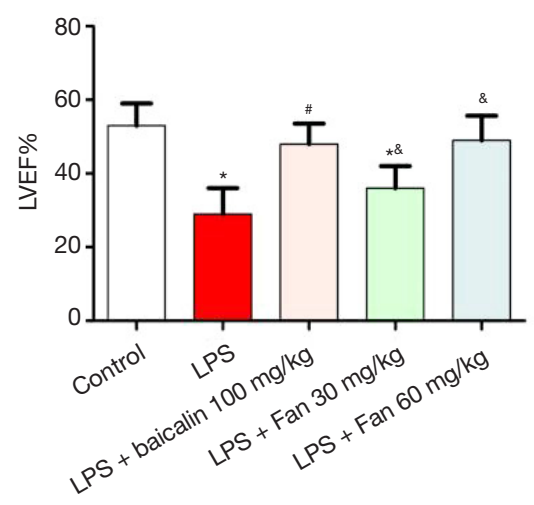

$E$

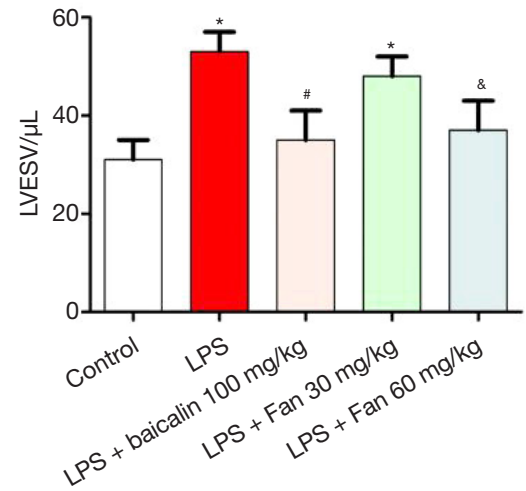

C

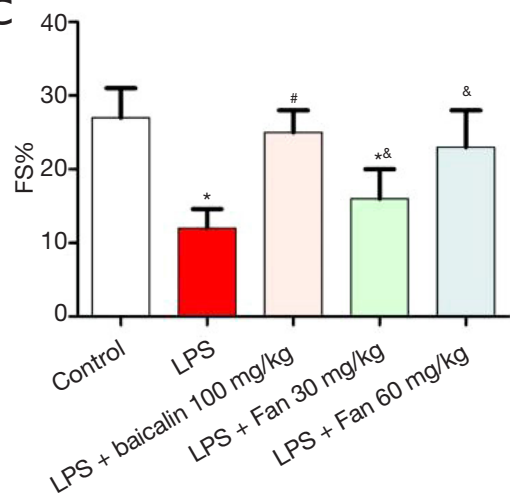

$\mathrm{F}$

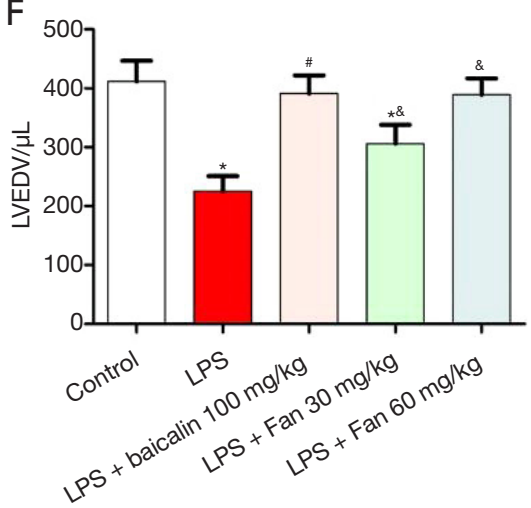

D 1.0

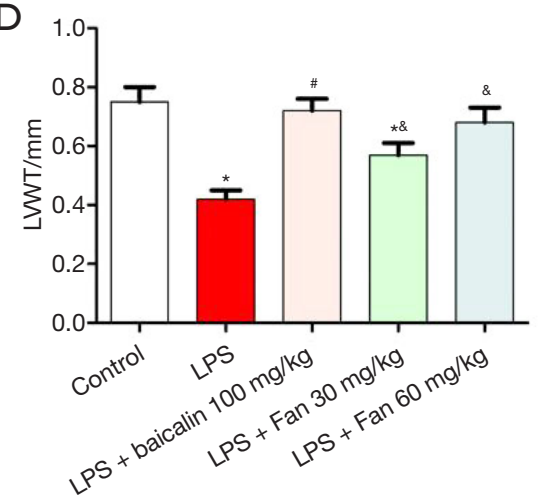

G

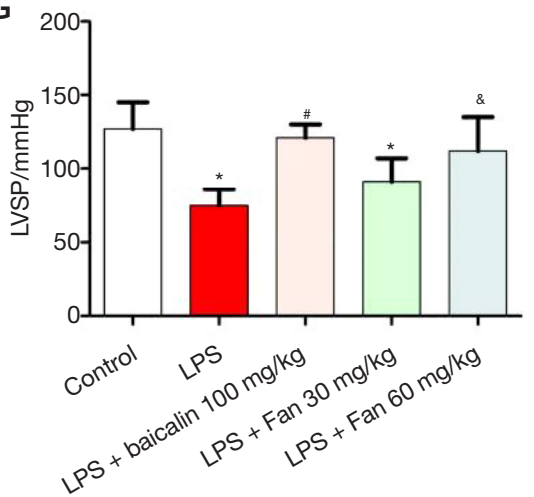

Figure 1 Effects of Fan on cardiac function in rats. After 12 hours of LPS treatment. (A) Cardiac cell infiltration was detected by HE staining, and representative images were taken under 400× magnification. (B) LVEF, (C) FS, (D) LVWT, (E) LVESV, (F) LVEDV, and (G) LVSP were assessed by echocardiography. Data are presented as the mean $\pm \mathrm{SD}(\mathrm{n}=10)$. * $\mathrm{P}<0.05$ versus the control group; ${ }^{\#, \&}$, $\mathrm{P}<0.05$ versus the LPS group. All independent experiments were repeated at least three times. Fan, Fangchinoline; LVEF, left ventricular ejection fraction; SD, standard deviation; FS, fractional shortening; LVWT, left ventricular wall thickness; LVESV, left ventricular end-systolic volume; LVEDV, left ventricular end-diastolic volume; LVSP, left ventricular systolic pressure; LPS, lipopolysaccharide.

inflammatory cytokines were significantly down-regulated, compared with the LPS group $(\mathrm{P}<0.05)$.

\section{Fan impaired the activity of NF- $\mathrm{KB}$ and ERK1/2 in LPS- induced rats}

To investigate the anti-inflammatory effects of Fan, the phosphorylation levels of NF- $\mathrm{KB}$ p 65 and ERK1/2 were examined by Western blot. The results revealed that LPS significantly up-regulated the protein levels of p65
(Figure 5A) and ERK1/2 (Figure 5B), compared with the control group $(\mathrm{P}<0.05)$. In contrast, Fan and Baicalin markedly inhibited the phosphorylation of p65 (Figure $5 A$ ) and ERK1/2 (Figure 5B) $(\mathrm{P}<0.05)$. Additionally, as phosphorylated p65 enters into the nucleus, we also examined the nuclear positive expression of $\mathrm{p} 65$ by IHC assay. As shown in Figure 5C, the level of p65 in the nucleus significantly increased after LPS treatment, compared with control group $(\mathrm{P}<0.05)$. However, Fan and Baicalin treatment remarkably inhibited the nuclear expression of 
A

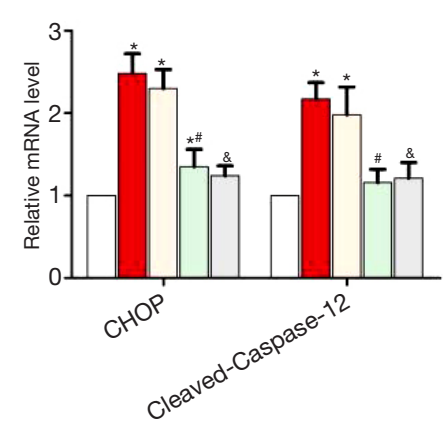

C

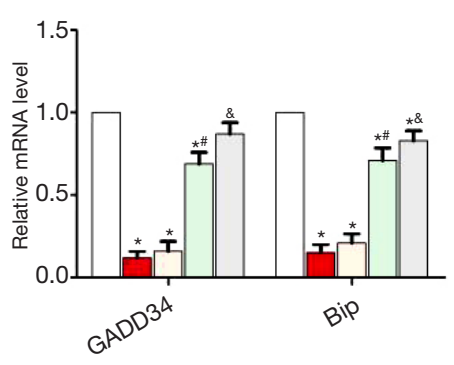

B
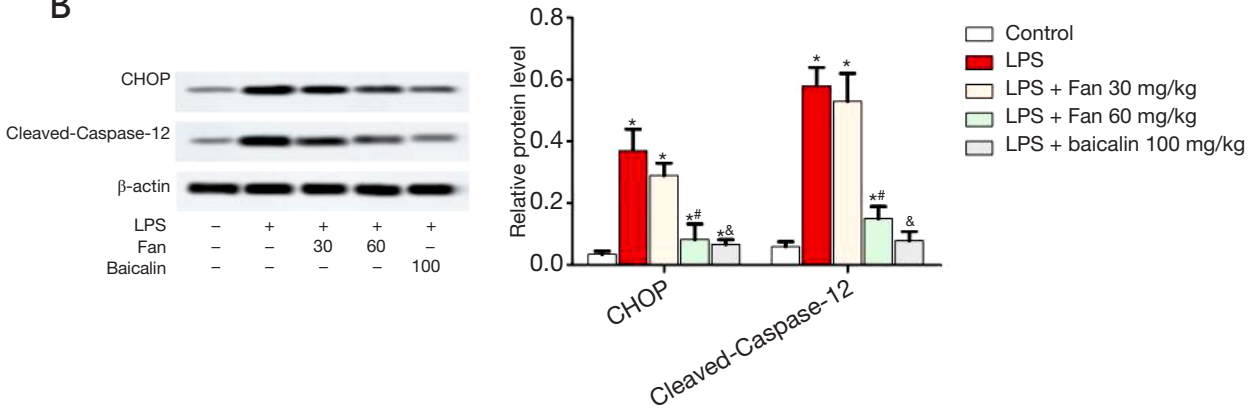

D
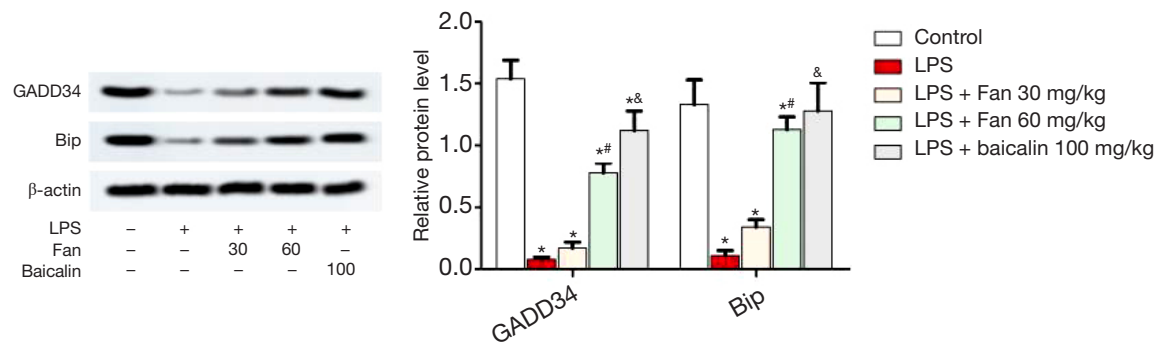

Figure 2 Effects of fan on ER stress-induced apoptosis. (A) The mRNA levels of CHOP and cleaved caspase-12 were examined by qRTPCR. (B) The protein levels of CHOP and cleaved caspase-12 were examined by western blot. (C) The mRNA levels of GADD34 and Bip were examined by qRT-PCR. (D) The protein levels of GADD34 and Bip were examined by western blot. Data are presented as the mean $\pm \mathrm{SD}(\mathrm{n}=10)$. *, $\mathrm{P}<0.05$ versus the control group; ${ }^{*, \&}, \mathrm{P}<0.05$ versus the LPS group. All independent experiments were repeated at least three times. ER, endoplasmic reticulum; qRT-PCR, quantitative reverse transcription-polymerase chain reaction; SD, standard deviation; LPS, lipopolysaccharide.

p65 in comparison with the LPS group $(\mathrm{P}<0.05)$.

\section{Fan attenuated endotoxemia through inbibiting $N F-\kappa B$ and ERK1/2 Pathway}

To understand the molecular mechanism underlying increased proliferation, we added the NF- $\kappa \mathrm{B}$ inhibitor pyrrolidine dithiocarbamate (PDTC) and ERK1/2 inhibitor U0126 into the jugular vein of rats and the rats grouped as below: control group, LPS group, Fan $(60 \mathrm{mg} / \mathrm{kg})$ group, PDTC $(100 \mu \mathrm{M})$ group, and U0126 $(10 \mu \mathrm{M})$ group. As shown in Figure 6 A,B,C, the phosphorylation levels of NF$\kappa \mathrm{B}$ p65 and ERK1/2 were decreased by PDTC, U0126 or Fan $(\mathrm{P}<0.05)$. In addition, PDTC, U0126 or Fan alone treatment reduced the levels of cleaved-Capase3, CHOP and, IL-1 $\beta$, compared with LPS group (Figure $6 D, E, F, G$ ).

\section{Discussion}

Sepsis is an organ dysfunction syndrome attributable to abnormal immune and inflammatory response to infection (23). It usually occurs following acute and systemic injury, resulting in acute organ dysfunction, such as myocardial damage and cardiac dysfunction, and even death. Therefore, more effective treatments for preventing cardiac dysfunction in sepsis urgently need to be discovered. Likewise, sepsis-induced cardiac dysfunction is characterized by LV systolic dysfunction, diastolic dysfunction, and right ventricular diastolic dysfunction $(24,25)$. In echocardiography, EF and FS are widely used as alternative markers of cardiac contractility and cardiac function. Zeng et al. reported that high doses of adenosine could significantly improve LVEF and LVFS in LPSinduced septic rats (6). In this study, we first assessed the index effect of LVEF and LVFS in LPS-induced rats by echocardiography. Our data revealed that LPS aggravated LVEF and LVFS, whereas Fan improved cardiac dysfunction. Moreover, Fan significantly decreased LVWT, LVEDV, and LVSP, but increased LVESV. All of our data indicated that Fan had a protective effect for cardiac function in rats with sepsis.

ER stress induction is caused by excessive protein 

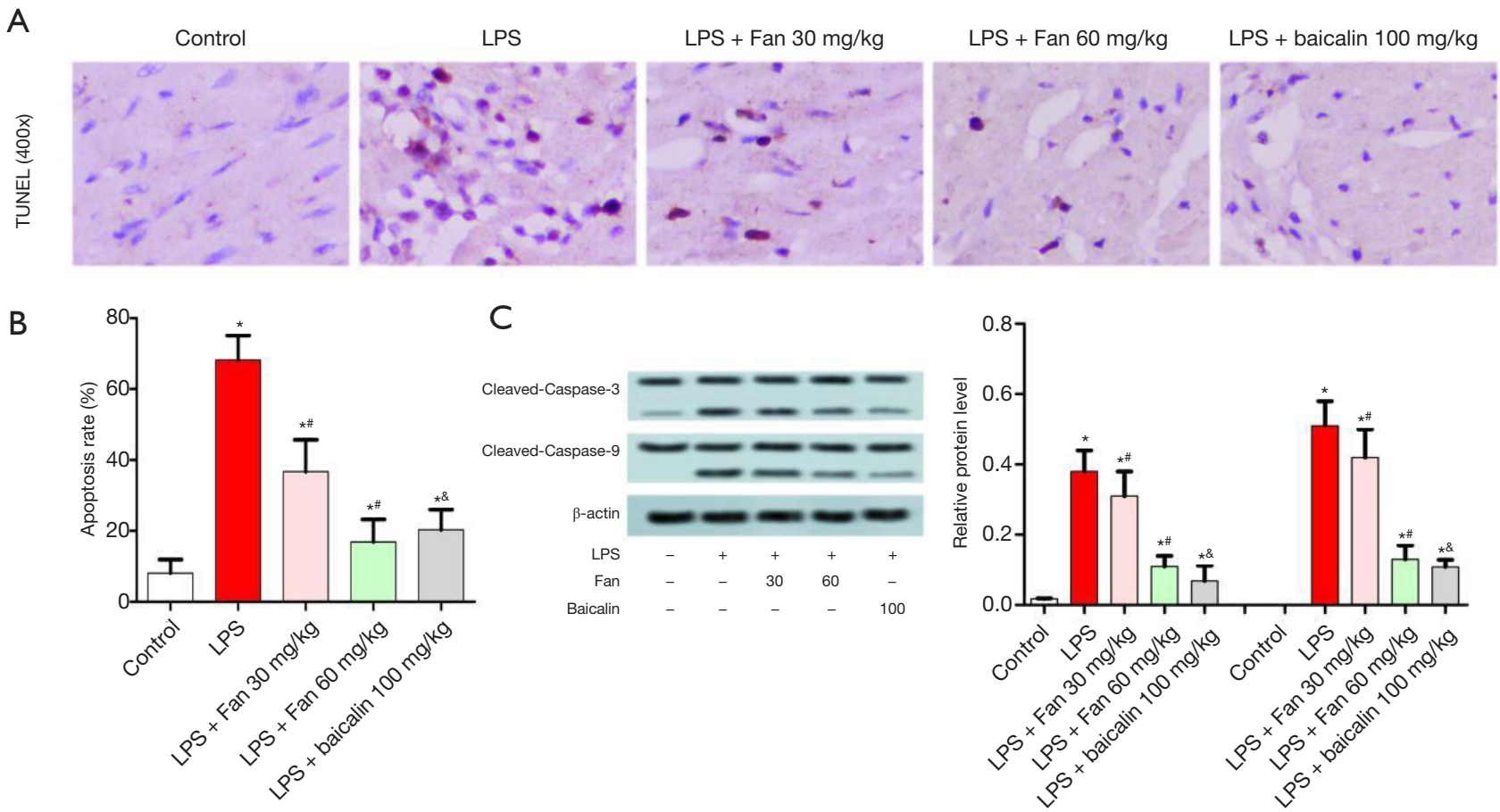

D
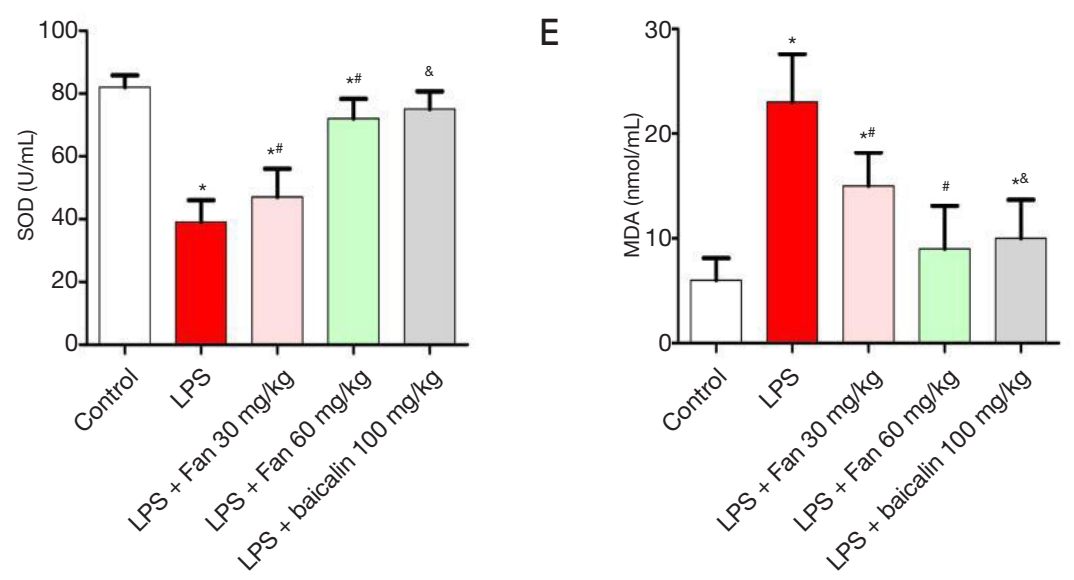

Figure 3 Effects of Fan on cardiac apoptosis and oxidative stress. (A,B) The tissue apoptosis of myocardial tissues was examined by TUNEL staining, and representative images were taken under 400× magnification. (C) The protein levels of Caspase-3, and Caspase-9 were examined by western blot. (D) The serum levels of SOD, and MDA were detected by ELISA assay. Data are presented as the mean \pm SD ( $\mathrm{n}=10$ ). *, $\mathrm{P}<0.05$ versus the control group; ${ }^{\#, \&}, \mathrm{P}<0.05$ versus the LPS group. All independent experiments were repeated at least three times. Fan, Fangchinoline; HE, hematoxylin and eosin; qRT-PCR, quantitative reverse transcription-polymerase chain reaction; SD, standard deviation; LPS, lipopolysaccharide.

misfolding or the accumulation of excess proteins (26). During ER stress, Golgi activation of ATF-6 and PERKmediated phosphorylation of eukaryotic translation initiation factor 2 (eIF $2 \alpha$ ) caused the overexpression of the chaperone gene GRP78/BiP, which promotes growth arrest and translation of $\mathrm{CHOP}$ and GADD34. Nakamura et al. reported CHOP to be the main effector of ER stressmediated apoptosis, and that loss or silencing of CHOP reduced macrophage apoptosis in vitro and in vivo (27). Excessive ER stress up-regulates the expression of CHOP and caspase-12 and promotes apoptosis of cardiomyocytes $(28,29)$. Therefore, in this study, we analyzed the expression 
A

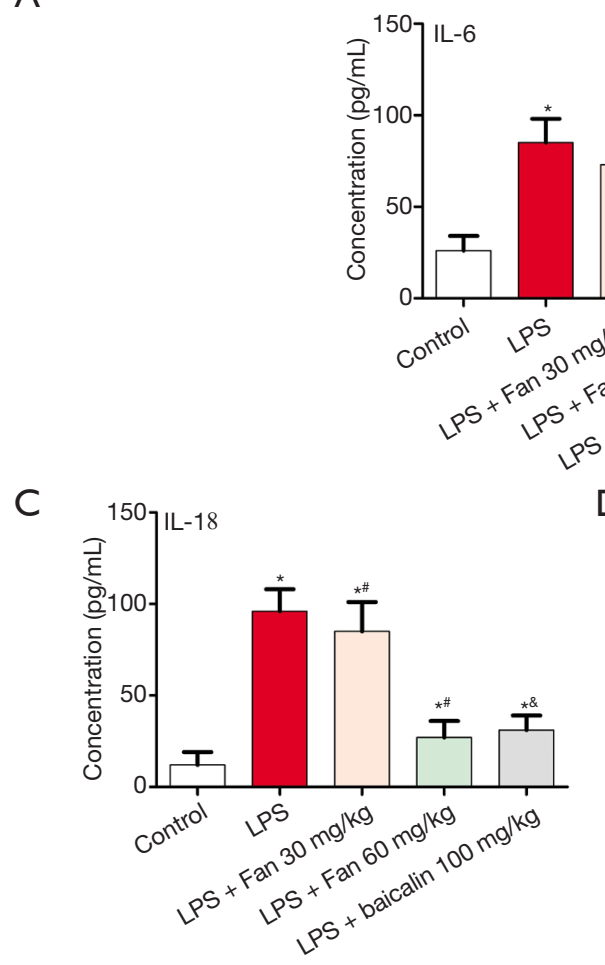

B

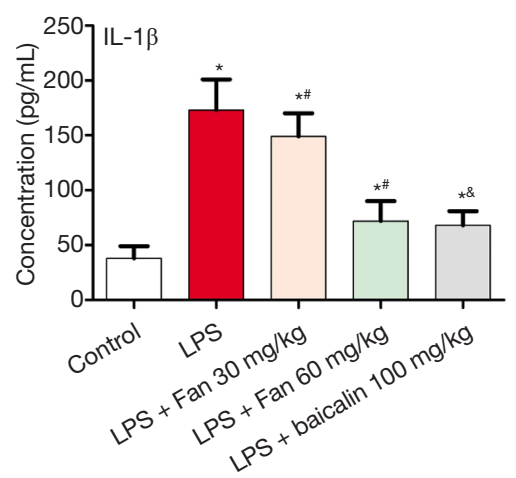

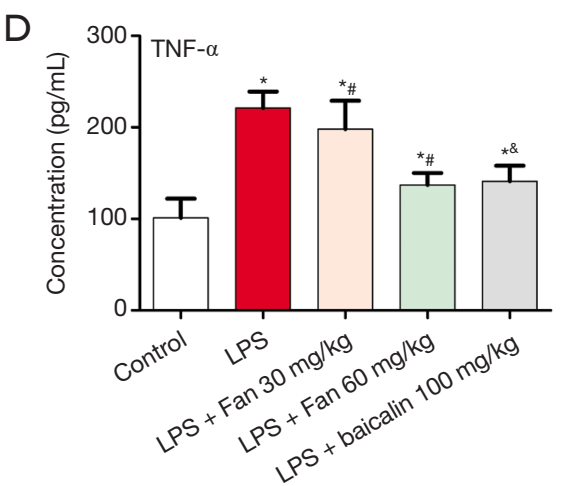

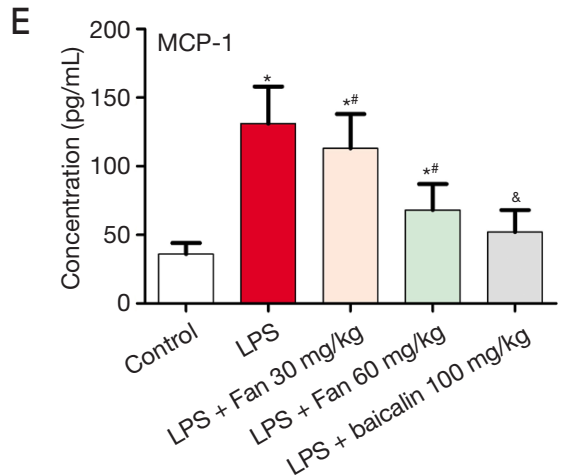

Figure 4 Fan inhibited LPS-induced inflammatory response. The levels of IL-6 (A), IL-1 $\beta$ (B), IL-18 (C), TNF- $\alpha$ (D), and MCP-1 (E) were measured by ELISA assay. Data are presented as mean $\pm S D(n=10)$. ${ }^{*}, \mathrm{~s}, \mathrm{P}<0.05$ versus the control group; ${ }^{\#}, \mathrm{P}<0.05$ versus the LPS group. All independent experiments were repeated at least three times. LPS, lipopolysaccharide; IL, interleukin; TNF- $\alpha$, tumor necrosis factor- $\alpha$; MCP-1, monocyte chemotactic protein-1; ELISA, enzyme-linked immunosorbent assay; SD, standard deviation.

levels of CHOP and caspase-12, and found that LPS greatly up-regulated $\mathrm{CHOP}$ and caspase-12 expression. Moreover, Cardiomyocyte apoptosis plays an important role in the pathogenesis of sepsis (30). LPS has been reported to up-regulate the levels of Bax, caspase-3, and caspase-9, and down-regulate the levels of anti-apoptotic protein Bcl-2 (31). A previous study demonstrated that Fan significantly increased cleavage of caspase- 3 in bladder cancer cells (16). Furthermore, Fan significantly attenuated the altered expressions of LC3, Bcl-2, caspase-3, BMP2, and Beclin-1, and reduced osteoblast apoptosis (20). Similarly, in our study, Fan significantly decreased caspase-3 and caspase-9 expression at the mRNA and protein levels. This suggests that Fan may suppress cardiac apoptosis via ER stress in LPS-treated rats.

Inflammation is well known to be a defense mechanism, which plays a critical role in sepsis-induced cardiac dysfunction. LPS has been observed to activate the inflammatory response of mononuclear cells and macrophages, causing many pathophysiological phenomena, including the release of a battery of proinflammatory factors, such as IL-1 $\beta$, TNF- $\alpha$, IL-6, and MCP-1 $(12,13)$. Among the inflammatory cytokines, IL-1 $\beta$ is a potent proinflammatory mediator in many immune responses. $\mathrm{Li}$ et al. suggested that LPS significantly increased the serum and myocardial levels of TNF- $\alpha$, MCP-1, and HMGB1 by inducing the secretion of IL-1 $\beta$, which further amplified inflammation in heart (32). In the present study, LPS strongly up-regulated the protein levels of MCP- 1 , TNF- $\alpha$, and IL- $1 \beta$, which is consistent with previous results. This finding suggests that LPS increases the expression of MCP-1 and TNF- $\alpha$ by elevating the level of IL-1 $\beta$. Furthermore, Liu $e t$ al. reported that Fan inhibited LPS-induced IL-1 $\beta$ release at both the cellular and protein levels (33), while another study showed that Fan significantly attenuated LPS-induced cardiac dysfunction and reduced the release of inflammatory cytokines such as VEGF, IL-6, IL-1b, and TNF- $\alpha$ in the retina (31). Subsequently, we examined the levels of MCP-1, TNF- $\alpha$, IL-1 $\beta$, IL-18 and IL-6. The results confirmed that the Fan-treated mice showed 
A

B
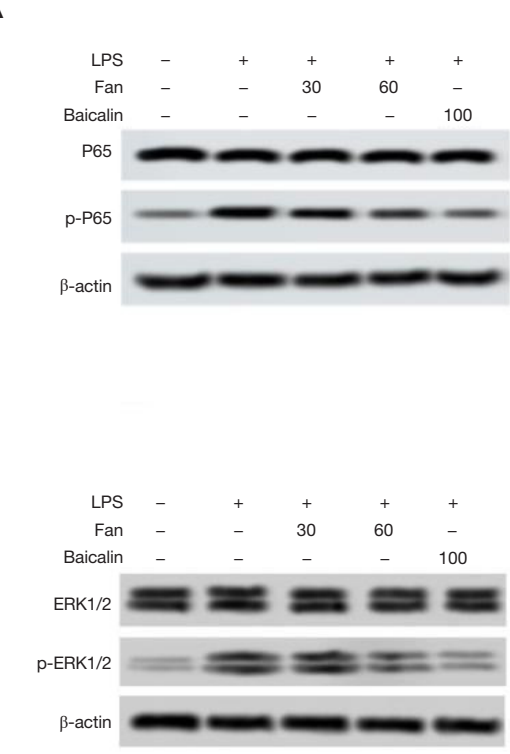
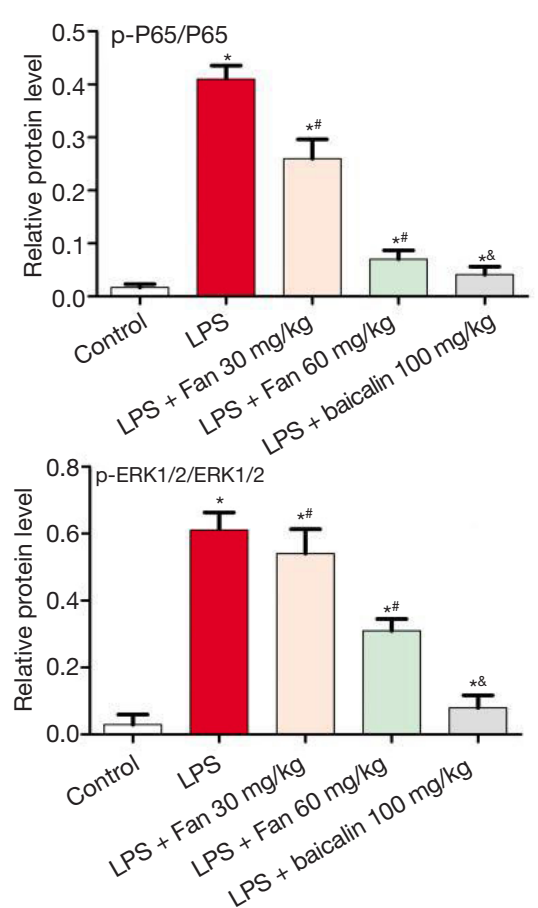

C
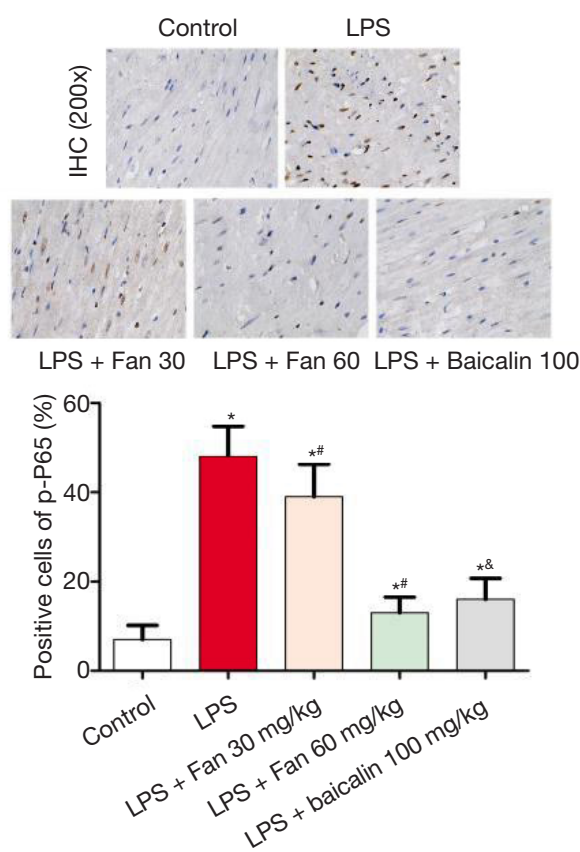

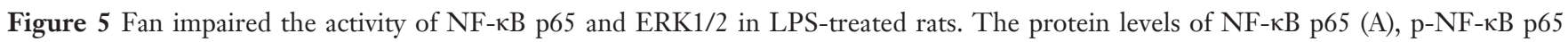
(A), ERK1/2 (B), and p-ERK1/2 (B) were measured by western blot. The nuclear positive level of NF-kB p65 (C) was measured by IHC assay, and representative images were taken under $200 \times$ magnification. Data are presented as mean $\pm S D(n=10)$. *,$P<0.05$ versus the control group; ${ }^{\#, \&}, \mathrm{P}<0.05$ versus the LPS group. All independent experiments were repeated at least three times. LPS, lipopolysaccharide; NF- $\mathrm{kB}$, nuclear factor- $\mathrm{\kappa B}$; IHC, immunohistochemistry; SD, standard deviation.

significantly reduced MCP-1, TNF- $\alpha$, IL- $1 \beta$, IL-18, and IL-6 expression. Taken together, these results suggested that Fan regulated LPS-mediated inflammatory cytokines to attenuate cardiac dysfunction in rats.

Acute cardiac dysfunction cause rupture, swelling or death of myocardial cell membrane. It can also seriously disrupt the oxidation and antioxidant balance in vivo (26). The rats were exposed to oxidative stress after injection of LPS, characterized by increased production of ROS and lipid peroxidation products, such as MDA (34). In this study, current available data found that Fan injection decreased MDA, simultaneously increased SOD activity. There is extensive evidence that LPS can activate multiple inflammatory pathways including NF- $\kappa \mathrm{B}$ and MAPKs pathways (35). NF-kB signaling plays an important role in the expression of a series of inflammatory cytokines (36). LPS induces myocardial inflammation (36) and apoptosis (37) by activating NF- $\kappa \mathrm{B}$ signaling pathways. Our study showed that the elevated p65 phosphorylation caused by LPS could be reversed by Fan, or PDTC, which suggested that Fan may attenuate LPS-induced cardiac dysfunction through inhibiting p65 activation and downstream cytokines production of cleaved-Capase 3 and, IL-1 $\beta$. Moreover, we found that Pae treatment significantly reduced Akt phosphorylation in LPS treated mice. Studies have shown that LPS induction increased ERK1/2 phosphorylation of cardiomyopathy (38). But, an in-depth study should be conducted to confirm this. Taken together, these results indicated that Fan can protect against LPS-induced cardiac dysfunction by inhibiting ERK1/2 and NF- $\mathrm{KB}$ pathway. In conclusion, our study demonstrated the protective role of Fan on LPS-induced cardiac dysfunction, which is mediated via the ERK1/2 and NF- $\mathrm{kB}$ pathways. Furthermore, Fan inhibited myocardial apoptosis following ER stress, and attenuated the myocardial inflammatory response and oxidative stress. Therefore, Fan could be a candidate for the treatment of cardiac dysfunction. 


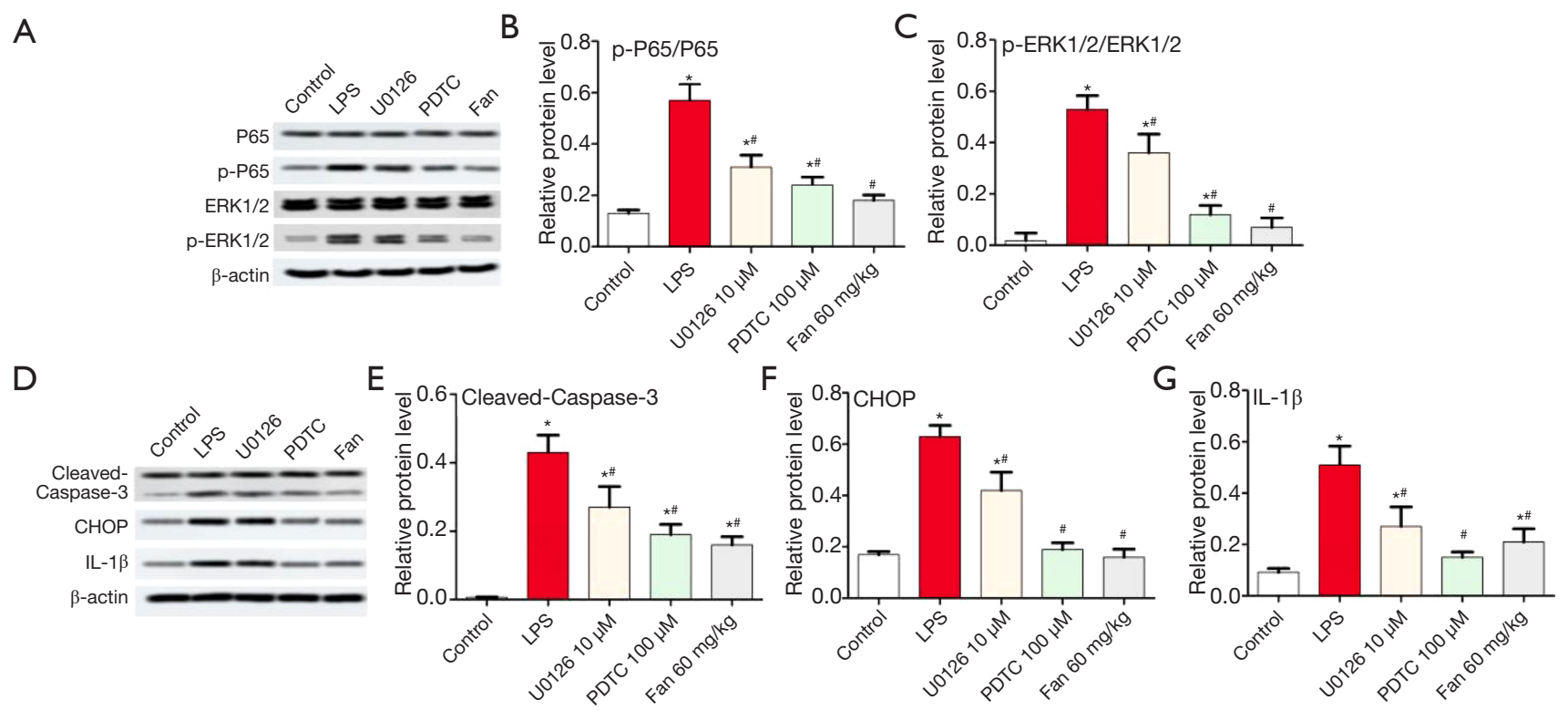

Figure 6 Fan attenuated endotoxemia through inhibiting NF- $\kappa$ B and ERK1/2 Pathway. (A,B,C,D,E,F,G) After adding NF- $\kappa B$ inhibitor PDTC and ERK1/2 inhibitor U0126, the protein levels of P65, p-P65, ERK1/2, p-ERK1/2, cleaved-Csapase3, CHOP, and IL-1 $\beta$ were examined by WB. Data are presented as mean $\pm \mathrm{SD}(\mathrm{n}=8)$. *, $\mathrm{P}<0.05$ versus the control group; *, $\mathrm{P}<0.05$ versus the LPS group. All independent experiments were repeated at least three times. LPS, lipopolysaccharide; PDTC, pyrrolidine dithiocarbamate; NF- $\kappa \mathrm{B}$, nuclear factor- $\kappa \mathrm{B}$; IHC, immunohistochemistry; SD, standard deviation.

\section{Acknowledgments}

Funding: None.

\section{Footnote}

Reporting Checklist: The authors have completed the ARRIVE reporting checklist. Available at http://dx.doi. org/10.21037/atm-20-5669

Data Sharing Statement: Available at http://dx.doi. org/10.21037/atm-20-5669

Conflicts of Interest: All authors have completed the ICMJE uniform disclosure form (available at http://dx.doi. org/10.21037/atm-20-5669). The authors have no conflicts of interest to declare.

Ethical Statement: The authors are accountable for all aspects of the work in ensuring that questions related to the accuracy or integrity of any part of the work are appropriately investigated and resolved. The study was approved by the Research Ethics Committee of Xinxiang Central Hospital (ZXYY-KY-0058), and were conducted according to the published Guide for the Care and Use of Laboratory Animals (National Academies Press, 2011).

Open Access Statement: This is an Open Access article distributed in accordance with the Creative Commons Attribution-NonCommercial-NoDerivs 4.0 International License (CC BY-NC-ND 4.0), which permits the noncommercial replication and distribution of the article with the strict proviso that no changes or edits are made and the original work is properly cited (including links to both the formal publication through the relevant DOI and the license). See: https://creativecommons.org/licenses/by-nc-nd/4.0/.

\section{References}

1. Zanotti-Cavazzoni SL, Hollenberg SM. Cardiac dysfunction in severe sepsis and septic shock. Curr Opin Crit Care 2009; 15:392-7.

2. Shahreyar M, Fahhoum R, Akinseye O, et al. Severe sepsis and cardiac arrhythmias. Ann Transl Med 2018;6:6.

3. Turner KL, Moore LJ, Todd SR, et al. Identification of cardiac dysfunction in sepsis with B-type natriuretic peptide. J Am Coll Surg 2011;213:139-46.

4. Charpentier J, Luyt CE, Fulla Y, et al. Brain natriuretic 
peptide: A marker of myocardial dysfunction and prognosis during severe sepsis. Crit Care Med 2004;32:660-5.

5. Landesberg G, Levin PD, Gilon D, et al. Myocardial dysfunction in severe sepsis and septic shock: No correlation with inflammatory cytokines in real-life clinical setting. Chest 2015;148:93-102.

6. Zeng M, Zhang B, Li B, et al. Adenosine attenuates LPSinduced cardiac dysfunction by inhibition of mitochondrial function via the ER pathway. Evid Based Complement Alternat Med. 2019;2019:1832025.

7. Sanfilippo F, Corredor C, Fletcher N, et al. Left ventricular systolic function evaluated by strain echocardiography and relationship with mortality in patients with severe sepsis or septic shock: a systematic review and meta-analysis. Crit Care 2018;22:183.

8. Lewis AJ, Seymour CW, Rosengart MR. Current murine models of sepsis. Surg Infect (Larchmt) 2016;17:385-93.

9. Rudiger A, Singer M. Mechanisms of sepsis-induced cardiac dysfunction. Crit Care Med 2007;35:1599-608.

10. Annane D, Bellissant E, Cavaillon JM. Septic shock. Lancet 2005;365:63-78.

11. Chao CN, Lo JF, Khan FB, et al. Tid1-S attenuates LPSinduced cardiac hypertrophy and apoptosis through ER-a mediated modulation of $\mathrm{p}-\mathrm{PI} 3 \mathrm{~K} / \mathrm{p}$-Akt signaling cascade. J Cell Biochem 2019;120:16703-10.

12. Zhai J, Guo Y. Paeoniflorin attenuates cardiac dysfunction in endotoxemic mice via the inhibition of nuclear factor$\kappa B$. Biomed Pharmacother 2016;80:200-6.

13. Tocmo R, Parkin K. S-Alk(en)ylmercaptocysteine suppresses LPS-induced pro-inflammatory responses in murine macrophages through inhibition of $\mathrm{NF}-\kappa \mathrm{B}$ pathway and modulation of thiol redox status. Free Radic Biol Med 2018;129:548-58.

14. Huang GJ, Deng JS, Chen CC, et al. Methanol extract of Antrodia camphorata protects against lipopolysaccharideinduced acute lung injury by suppressing NF-кB and MAPK pathways in mice. J Agric Food Chem 2014;62:5321-9.

15. Wang X, Zingarelli B, O'Connor M, et al. Overexpression of Hsp20 prevents endotoxin-induced myocardial dysfunction and apoptosis via inhibition of NF-kappaB activation. J Mol Cell Cardiol 2009;47:382-90.

16. Fan B, Zhang XY, Ma YL, et al. Fangchinoline induces apoptosis, autophagy and energetic impairment in bladder cancer. Cell Physiol Biochem 2017;43:1003-11.

17. Luo X, Peng JM, Su LD, et al. Fangchinoline inhibits the proliferation of SPC-A-1 lung cancer cells by blocking cell cycle progression. Exp Ther Med 2016;11:613-8.
18. Wang N, Pan W, Zhu M, et al. Fangchinoline induces autophagic cell death via p53strin2/AMPK signalling in human hepatocellular carcinoma cells. Br J Pharmacol 2011;164:731-42.

19. Gülçin I, Elias R, Gepdiremen A, et al. Antioxidant activity of bisbenzylisoquinoline alkaloids from Stephania rotunda: Cepharanthine and fangchinoline. J Enzyme Inhib Med Chem 2010;25:44-53.

20. Zhu W, Ding W, Shang X, et al. Fangchinoline Promotes Autophagy and Inhibits Apoptosis in Osteoporotic Rats. Med Sci Monit 2019;25:324-32.

21. Bai J, Wang Q, Qi J, et al. Promoting effect of baicalin on nitric oxide production in CMECs via activating the PI3KAKT-eNOS pathway attenuates myocardial ischemiareperfusion injury. Phytomedicine 2019;63:153035

22. Han D, Xia S, Zhu H, et al. Fangchinoline ameliorates the expressions of angiogenic molecule in cerebral ischemia induced neuronal degeneration in neonatal rats. Transl Neurosci 2018;9:117-22.

23. Fernandes CJ, de Assuncao MS. Myocardial dysfunction in sepsis: a large, unsolved puzzle. Crit Care Res Pract 2012;2012:896430.

24. Birati E.Y, Mathelier H, Molina M. Comparison of causes of death after heart transplantation in patients with left ventricular ejection fractions $\leq 35 \%$ versus $>35$. Am J Cardiol 2016;117:1322-6.

25. Vallabhajosyula S, Kumar M, Pandompatam G, et al. Prognostic impact of isolated right ventricular dysfunction in sepsis and septic shock: an 8-year historical cohort study. Ann Intensive Care 2017;7:94.

26. McDuffee AT, Senisterra G, Huntley S, et al. Proteins containing non-native disulfide bonds generated by oxidative stress can act as signals for the induction of the heat shock response. J Cell Physiol 1997;171:143-51.

27. Nakamura T, Furuhashi M, Li P, et al. Doublestranded RNA-dependent protein kinase links pathogen sensing with stress and metabolic homeostasis. Cell 2010;140:338-48.

28. Durante $W$. Targeting endoplasmic reticulum stress in hypoxia-induced cardiac injury. Vascul Pharmacol 2016;83:1-3.

29. Choy KW, Murugan D, Mustafa MR. Natural products targeting ER stress pathway for the treatment of cardiovascular diseases. Pharmacol Res 2018.;132:119-29.

30. Bommhardt U, Chang KC, Swanson PE, et al. Akt decreases lymphocyte apoptosis and improves survival in sepsis. J Immunol 2004;172:7583-91.

31. Zeng M, Zhang L, Zhang B, et al. Chinese yam extract 
and adenosine attenuated LPS-induced cardiac dysfunction by inhibiting RAS and apoptosis via the ER-mediated activation of SHC/Ras/Raf1 pathway. Phytomedicine 2019;61:152857.

32. Li N, Zhou H, Wu HM, et al. STING-IRF3 contributes to lipopolysaccharide-induced cardiac dysfunction, inflammation, apoptosis and pyroptosis by activating NLRP3. Redox Biol 2019;24:101215.

33. Liu T, Zeng QX, Zhao XQ, et al. Synthesis and biological evaluation of fangchinoline derivatives as anti-Inflammatory agents through inactivation of inflammasome. Molecules 2019;24:1154.

34. Giralt M, Gasull T, Blanquez A., et al. Efect of endotoxin on rat serum, lung and liver lipid peroxidation and on tissue metallothionein levels. Revista Espanola de Fisiologia 1993;49:73-8.

35. Guo S, Jiang K, Wu H, et al. Magnoflorine ameliorates

Cite this article as: Chen H, Shi Z, Xing Y, Li X, Fu F. Fangchinoline attenuates cardiac dysfunction in rats with endotoxemia via the inhibition of ERK1/2 and NF- $\kappa \mathrm{B}$ p 65 phosphorylation. Ann Transl Med 2020;8(18):1167. doi: 10.21037/atm-20-5669 lipopolysaccharide- induced acute lung injury via suppressing NF- B and MAPK activation. Front Pharmacol 2018; 9:982.

36. Kawai T, Akira S. Signaling to NF- B by Toll-like receptors. Trends Mol Med 2007;13: 460-9.

37. Tien YC, Lin JY, Lai CH, et al. Carthamus tinctorius L. prevents LPS-induced TNFalpha signaling activation and cell apoptosis through JNK1/2-NFkappaB pathway inhibition in H9c2 cardiomyoblast cells. J Ethnopharmacol 2010;130: 505-13.

38. Kaminska B. MAPK signalling pathways as molecular targets for anti-inflammatory therapy--from molecular mechanisms to therapeutic benefits. Biochim Biophys Acta 2005; 1754:253-62.

(English Language Editor: J. Reynolds) 